\title{
EMPLOYEE ACCEPTABILITY OF WEARABLE MENTAL WORKLOAD MONITORING IN INDUSTRY 4.0: A PILOT STUDY ON MOTIVATIONAL AND CONTEXTUAL FRAMING
}

\author{
Van Acker, Bram B. (1,2); Conradie, Peter (1,2); Vlerick, Peter (1); Saldien, Jelle (1,2) \\ 1: Ghent University, Belgium; 2: Research group IMEC-MICT-Ghent University, Belgium
}

\begin{abstract}
As Industry 4.0 will greatly challenge employee mental workload (MWL), research on objective wearable MWL-monitoring is in high demand. However, numerous research lines validating such technology might become redundant when employees eventually object to its implementation. In a pilot study, we manipulated two ways in which employees might perceive MWL-monitoring initiatives. We found that framing the technology in terms of serving intrinsic goals (e.g., improving health) together with an autonomy-supportive context (e.g., allowing discussion) yields higher user acceptability when compared to framing in terms of extrinsic goals (e.g., increasing productivity) together with a controlling context (e.g., mandating use). User acceptability still panned out neutral in case of the former, however - feeding into our own and suggested future work.
\end{abstract}

Keywords: Wearable mental workload monitoring, Industry 4.0, Organizational processes, Technology, User acceptability

\section{Contact:}

Van Acker, Bram Boris

Ghent University

Industrial Systems Engineering

Belgium

bramb.vanacker@ugent.be 


\section{INTRODUCTION}

The consumer market for wearable health technology monitoring physiological data is booming (cf., Luo, 2015). Different research fields also investigate the modalities and boundary conditions for applying such technology and other originally clinical technology (cf., fNIRS, EEG) on the shop floor and in office environments to monitor employee's mental workload (cf., Young et al., 2014). In Industry 4.0 in particular, such physiological measurement could be integrated in a cyber-physical system allowing, for instance, assistive technology such as augmented reality and assistive robots to immediately adapt to operator MWL (D'Addona et al., 2018). Indeed, as the smart factory is taking shape through integrating advanced manufacturing techniques with digital infrastructures, the effects of the extensive flexibility expected from operators (Longo et al., 2017) on the mental processing could benefit from mental workload optimization as supported by objective MWL-monitoring.

Whereas consumers might wear health technology to improve one's personal health (cf., Rupp et al., 2016), implementation of such technology on the shop floor could as well be perceived by employees as a surveillance tool to control productivity in order to improve efficiency and profit. Both perceptions underlying the application of this technology might have differential effects on the employee acceptability of such technology however (cf., the coercive vs. caring distinction in workplace electronic surveillance, Watkins Allen et al., 2007). If acceptability, here defined as a prospective judgment about technology to be introduced (Vlassenroot et al., 2010), turns out to be low, reluctance to adopt the technology or low compliance in case of final implementation might be the result.

How employee perception about physiological mental workload monitoring and its effect on user acceptability pans out, we think, strongly depends - among other variables - on the motivational reasons for the implementation of the technology as perceived by the employees or as communicated by the company (the 'why' of wearable mental workload monitoring) and how the technology is (said or thought to be) contextualized by the company (the 'how' of wearable mental workload monitoring). In the current pilot study, we experimentally manipulated this 'motivational framing' and 'contextual framing', respectively, based on Vansteenkiste et al.'s (2004) experimental design rooted in Selfdetermination Theory (Ryan and Deci, 2000). Since the job flexibility expected from operators in the future Industry 4.0 urges for job optimization tools supported by various wearables, we believe that user acceptability of these wearables should be considered a major challenge for the future workplace.

\section{THEORETICAL BACKGROUND AND HYPOTHESES}

Highly effective organizations do not only centralize profit, but cultivate performance and employee motivation and wellness (Deci et al., 2017). Wearable MWL-monitoring is believed to be helpful in both. Wearable measures for mental workload estimation typically use bodily reactions such as heart rate, brain oxygenation, pupil dilation, sweat secretion, etc. (for an overview, see Young et al., 2014; Van Acker et al., 2018). Such measures capture personal data linking them to the work performed in order to optimize underload or overload thereby, i.a., avoiding risks to employee safety, improving efficiency, job satisfaction and wellbeing (Matthews, 2016; Wickens, 2017). Both the employer (cf., efficiency and profit) and the employee (cf., safety and health) could thus benefit from this technology. But what would both end-goals mean to its acceptability? Self-determination theory (Ryan and Deci, 2000) could help provide an answer.

\subsection{The 'Why' of MWL-monitoring: motivational framing}

Self-determination theory (Ryan and Deci, 2000) makes a distinction between intrinsic goals, being innately satisfying and pursued for the activity per se, and extrinsic goals, being instrumental in nature while strived after for contingent outcomes distinct from the activity itself. Examples of intrinsic goals are typically, e.g., personal growth and improving one's health (cf. Kasser and Ryan, 1996) or seizing learning opportunities and performing interesting work (Van den Broeck et al., 2011). Extrinsic goals then are for instance attaining wealth and image (cf., Kasser and Ryan, 1996) or job security and economic returns in the workplace (Van den Broeck et al., 2011). Intrinsic goal striving is found to outperform extrinsic goal pursuit on variables such as job engagement, organizational citizenship, mental health, persistence, involvement and, even more important for our research question, 
acceptance of organizational change (cf., Mitchell et al., 2012). SDT explains these differences based on the notion that intrinsic goals promote the human natural growth tendencies through the satisfaction of the basic psychological needs for autonomy, competence and relatedness (Deci and Ryan, 2000). Extrinsic goals do not cultivate these needs very well (Vansteenkiste et al., 2007), since they are instrumental in nature such as obtaining rewards and avoiding punishments (Mitchell et al., 2012). When introducing new technologies, organizations can communicate or employees can perceive its implementation as motivated by one or both goal types. MWL- monitoring could for instance help in achieving intrinsic goals such as creating meaningful jobs and a positive work environment. Contrarily, employees might as well fear and avoid punishment as a function of the extrinsic goal of safeguarding job security when thinking of MWL-monitoring as a measure or predictor of low productivity (Alder, 2001). Based on the findings that intrinsic goal pursuit is more positively related to job engagement and acceptance of organizational change, for example, and because they tap into the human natural growth tendencies, we could thus expect that MWL-monitoring should lead to higher employee acceptability when related to intrinsic goals. Still, the literature also provides suggestions to back the opposite. Sarpong and Rees (2014), interestingly, suggest that surveillance of employees ('big brother' in the workplace) can be perceived by employees as value neutral and a win-win situation, while Watkins Allen et al. (2007) also suggest that surveillance can be perceived as positive because it protects employees from lazy or incompetent coworkers. This contradiction thus presents a first call for empirical investigation. Apart from the goals of wearable MWL-monitoring also the way in which it is implemented should predict acceptability.

\subsection{The 'How' of MWL-monitoring: contextual framing}

When new technology is (said) to be introduced, mental, emotional and attitudinal states experienced on the job and being part of the 'employee psychological contract' (Sparrow, 2001) will inform the beliefs and perceptions held toward the conditions in which the technology will reside. These states involve, e.g., feelings of trust, perceived justice and perceived organizational support (Sparrow, 2001; see also Mitchell et al., 2012). and are typically promoted in an autonomysupportive work context, according to SDT - a context in which employee opinions and participation in decision making are appreciated and well considered. In the contrary case, a controlling organizational context pressures employees to do things (cf., Roche and Haar, 2013). Also autonomy-support shows to be positively related to job satisfaction and performance, work commitment, loyalty for the organization, lower turnover intentions (cf., Rigby and Ryan, 2018) and, interestingly, again adoption of organizational change (Oreg, 2006). The nature of the work context, we reason, will hence be of pivotal importance in case of wearable MWL-monitoring technology since personal data are captured and exactly trust, justice and support when processing and interpreting these data should predict employee acceptability.

A survey study on the challenges of introducing wearable sensors to the workplace revealed that privacy concerns among employees could be the highest adoption barrier for wearable sensors (Schall et al., 2018). When employees trust their superiors, feel that their superiors highly value justice and perceive their organization to provide opportunities to share opinions (cf., Deci et al., 2017), acceptability of wearable MWL-monitoring could be higher, as this setting might be perceived as one in which personal data will be treated with ethical care and employee's opinions will be genuinely considered.

Again, the literature contains some opposing suggestions however, as Venkatesh and Davis (2000) and Prasad and Agarwal (1997) in the tradition of the technology acceptance field proposed that mandating usage of a new system in the work environment could help employees overcome the momentum of initial use. Still, they point to potential negative effects on the long term and the personal data as acquired by the technology we investigate here might present a specific case. The existing literature that might predict employee acceptability as a function of the why and how thus both suggests contradictory outcomes. Still, we believe that the findings of the SDT-literature could help us hypothesize into one direction.

\subsection{Hypotheses}

Within the SDT research lines, Vansteenkiste et al. (2004) found that communicating intrinsic goal contents (community, personal growth, health) and autonomy-supportive contexts (e.g., "if you 
choose") can synergistically improve student's learning, performance and persistence (concerning new text material and new physical exercises), as compared to extrinsic goal contents (money, image) and controlling contexts (e.g., "you have to"). Based on these findings and on the foundations of Selfdetermination Theory (Ryan and Deci, 2000), we reasoned that also the implementation in the work environment of a wearable mental workload gauge might be communicated and implemented by companies - and might certainly be perceived by its employees (based on anecdotal information we obtained in studies applying such gauge) - as serving intrinsic goals and/or extrinsic goals, and might also be contextualized or perceived as autonomy-supportive and/or as controlling. Specifically, we reckoned that the user acceptability of such wearable technology could strongly depend on framing or perceiving the use of it in terms of:

- Meaningful work creation, personal health improvement and taking care of a positive work environment (i.e., intrinsic goals)

- Efficiency improvement, productivity improvement and profit gains (i.e., extrinsic goals)

- Providing a choice to wear it and discussing its output in dialogue with the employees (i.e., an autonomy-supportive context)

- Making the device mandatory and not discussing the data with employees (e.g., a controlling context)

How then this Motivational framing (i.e., intrinsic vs. extrinsic) and Contextual framing (i.e., autonomy-supportive vs. controlling) affects user acceptability of a wearable physiological mental workload gauge, is rather contradictory when we look at the relevant literature presented above. Because the work of Prasad and Agarwal (1997), Sarpong and Rees (2014), and Venkatesh and Davis (2000) and Watkins Allen et al. (2007), moreover, do not experimentally manipulate mandating the use of new technology, nor does it take into account motivational framing contrary to the work of Vansteenkiste et al. (2004) that we build on, we hypothesized:

1. "Intrinsic goal framing + Autonomy-support framing (Condition 2) will yield higher scores on User Acceptability, as compared to Extrinsic goal framing + Controlling framing (Condition 1)."

2. "Technology Readiness will moderate this effect, in that people scoring higher on the Technology Readiness will show a higher User Acceptability."

Since this was a pilot study - in which we did not know the final sample size and first wanted to explore effects of the two extremes - we thus designed two conditions (and not 4 as in Vansteenkiste et al., 2004) taking together one way of framing the motivational rationale and the context of application. These two conditions constituted of the combinations revealing the synergetic effect in Vansteenkiste et al. (2004). We did so, again in line with these authors, with a survey study in which we presented a letter to one group of participants in which the motivation and context of wearable mental workload monitoring was framed as serving intrinsic goals and autonomy supportive and to the other group as serving extrinsic goals and controlling. In this way, we manipulated perceptions that employees might have in real-life when gathering information about, or merely hearing about, MWL-monitoring.

We did not have hypotheses on moderating effects of our demographic variables age, gender and education level, since we did not have literature backing any directions on these variables. We did explore for their effects.

\section{METHODS}

We sent an online survey to a convenience sample of Dutch speaking Belgian working adults (thus not necessarily working in an industrial environment) in the context of an non-related undergrad methodology class on consumer product satisfaction. We did not perform a power analysis, since we did not know how much employed respondents would complete the survey. Eventually $N$ turned out to be 150 (59.3\% female, Age: $M=48.77, S D=9.63)$. Participants were randomly assigned to one of two conditions (Condition 1, $n=76$, Condition 2, $n=74$ ). 
After the demographics part, respondents were presented one of two versions of a letter and were asked to imagine receiving this letter from their company. In these letters (see Appendix), 'their company' expressed to consider implementing wearable mental workload monitoring technology on the shop floor. This technology was described equally over both conditions as a system collecting physiological data and that 'the gauge worn on the body can indicate how hard someone is exerting mental effort based on bodily reactions such as heart beat, sweating and the gaze of the eyes' (translated from Dutch). Also what it does was written identically in both conditions, namely 'it registers how much mental effort is being exerted during work'. The letters however differed in terms of the extrinsic goal contents along with a controlling context in one letter (Condition 1) and intrinsic goal contents and an autonomysupportive context in the other (Condition 2). We manipulated these motivations and context by framing the reasons for implementing this mental workload technology as 'improving efficiency, productivity and profit' together with words such as 'have to wear' and so 'the company can redesign tasks'. In Condition 2 it was stated that the goal of the gauge is to 'create meaningful work, improve personal health and taking care of a positive work environment', this together with words such as 'in cooperation with the employees' and 'the company can learn' which tasks lead to mental overload. Manipulation with these words was based on words used in previous research on intrinsic and extrinsic goals (cf., Vansteenkiste et al., 2005) and in questionnaires gauging autonomy-supportive versus controlling contexts such as the Work Climate Questionnaire (Baard et al., 2000).

After reading one of these two letters, participants then completed questions forming a scale customized to measure User Acceptability and two open-ended questions. The items, measured on a 5point Likert scale, and open-ended questions were the following (translated from Dutch): 'I would support this idea', 'Why would you or why would you not?' (open-ended question), 'I would have confidence that my employer does not abuse my personal data measured with this gauge', 'I would wear such gauge', 'I find the reasons for this initiative just', 'I would recommend such gauge to my colleagues', 'My personal data measured with this gauge: May always stay anonymous' (at left anchor of scale), 'May always be linked to my name' (at right anchor of scale), 'Do you have any remarks or comments on this?' (open-ended question).

The questions were inspired on items from, e.g., Venkatesh and Davis (2000), extended with items measuring privacy concerns (based on Watkins Allen et al., 2007) and specific questions the authors designed. At the end of the questionnaire the respondents finally answered questions of the Technology Readiness Index scale (cf., Parasuraman and Colby, 2015).

\section{RESULTS}

Before our regression analysis, we calculated the reliability of our User Acceptability scale. Cronbach's alpha for the six items in our scale was high at $\alpha=0.93$. As a result, we calculated the average score. For Condition 1 the average was $1.87(n=75)$, while Condition 2 averaged $2.94(n=74)$.

Following this, we performed a linear regression. The results can be seen in Table 1 as Model 1a and 1b. Model 1a introduces our control variables: age, gender (male as reference category), higher education (no higher education as reference category) and TRI, while Model $1 \mathrm{~b}$ (extrinsic goal with controlling context condition as reference) introduces our experimental conditions.

We find no effect for either age or TRI, while gender $(B=0.58, p<0.01)$ and higher education $(B=$ $-0.60, p<0.01)$ did have a significant impact. In total, these control variables account for $10 \%$ of the total variance (Adjusted $R^{2}$ ) in acceptability scores, $F(4,145)=5.24, p<.001$. Subsequently, we introduce our categorical experimental condition in Model 1b. As a result, gender $(B=0.48, p<0.01)$, education $(B=-0.42, p<0.05)$ and our experimental condition significantly impacts User Acceptability $(B=1.02, p<0.001), F(5,144)=14.38, p<.001$. Overall, our results (see Table 1$)$ thus show that Intrinsic goal framing with Autonomy-supportive contextual framing significantly impacts User Acceptability. Both Model 1a and $1 \mathrm{~b}$ are statistically significant. Our experimental conditions, moreover, explain $20 \%$ incremental variance next to the control variables, so that in total Model $1 \mathrm{~b}$ explains $31 \%$ of variance in the dependent variable. Assessment of Variance Inflation Factor, which tests for multicollinearity finds no values higher than 1.01, suggesting no issues of multicollinearity. 
Table 1. Results of linear regression analysis on user acceptability. ${ }^{*} p<0.05 ;{ }^{* *} p<0.01$; ${ }^{* * *} p<0.001$.

\begin{tabular}{|l|c|c|}
\hline & Model 1a & Model 1b \\
\hline & $B($ S.E. $)$ & $B($ S.E. $)$ \\
\hline Control Variables & & $-0.01(0.01)$ \\
\hline Age & $-0.01(0.01)$ & $0.48^{* *}(0.15)$ \\
\hline Gender & $0.58^{* *}(0.18)$ & $-0.42^{*}(0.18)$ \\
\hline Education & $-0.60^{* *}(0.20)$ & $0.02(0.01)$ \\
\hline TRI & $0.03(0.02)$ & $1.02^{* * *}(0.15)$ \\
\hline Independent Variables & & $1.03^{*}(0.52)$ \\
\hline Condition & & \\
\hline Constant & $2.15^{* * *}(0.56)$ & 0.33 \\
\hline Explained variance & & 0.31 \\
\hline $\mathrm{R}^{2}$ & 0.13 & \\
\hline Adjusted $\mathrm{R}^{2}$ & 0.10 & \\
\hline
\end{tabular}

\section{DISCUSSION AND FUTURE WORK}

The motivation underlying research into, and final implementation of, wearable MWL-monitoring serves both performance and well-being goals. Employee acceptability is however still pivotal in achieving these goals. We reasoned that not only the content of the goals, but also the way in which they would be achieved by means of the technology should have differential effects on employee acceptability. The available literature points at both positive and negative outcomes of both motivations and two types of contexts we put forward. We therefore wanted to empirically test how employees would relate to these motivations and contexts by designing a pilot study presenting employees with a letter describing wearable MWL-monitoring in two ways. We found that framing the technology in terms of serving the intrinsic goals of creating meaningful work, improving personal health and taking care of a positive work environment together with indications of an autonomysupportive context of implementation yielded higher scores on user acceptability, as compared to framing the technology as serving the extrinsic goals of improving efficiency, productivity and profit together with indications of a controlling context of implementation.

User acceptability was measured with items gauging idea support, trusting the employer with the personal data, willingness to wear the technology, finding the reasons for it just, recommending the technology to a colleague and having no problems relating the data to one's name - all of which showing significant differences. Contrary to one of our hypotheses, technology readiness did not have an effect, while higher education and gender did yield an effect, in that higher educated people showed lower acceptability and female respondents showed higher acceptability rates. In total, these variables and our condition explained 31 percent (Adjusted $R^{2}=0.31$ ) of the total variance in User Acceptability.

The current pilot study nevertheless contained important caveats. First, we only implemented two conditions, so that only two extreme ways of framing the technology were scrutinized. Secondly, we used a convenience sample of people being employed and are hence not able to generalize to employees working in the future Industry 4.0 work context. Third, our letters did not provide contextual information on how the personal data would be treated. This is an important shortcoming, since safeguarding anonymity of data and for instance interpreting data only with scores aggregated over multiple employees could mean that more people might support the technology, perhaps even in case of serving the extrinsic goals of productivity, efficiency and profit. Also when the technology would serve intrinsic goals in an autonomy-supportive context, data privacy and related concerns could yield higher scores on acceptability. It is therefore perhaps not surprising that the average score of the condition outperforming the other only shows to be neutral, i.e., $M=3.12$ on a 5-point Likert scale. Future research could thus do well in first entangling our proposed effects into more conditions to approximate more nuanced perceptions, gathering data from a more representative sample, providing contextual data and/or addressing important other contextual variables such as the 
respondent's current perceived organizational trust, organizational privacy management and how the results from the MWL-monitoring would be interpreted.

From our side, we have already built upon the current findings by designing and pre-registering a follow-up study exploring replicability of the effect found. We additionally included the respondent's currently perceived organizational trust as a control variable and we also combined intrinsic goal contents with controlling environments and vice versa, while including a fifth control condition providing no rationale nor contextual information at all ${ }^{1}$. In this follow-up study, we gathered data of 350 respondents, though again not from a sample of industrial operators for practical reasons. Preliminary results suggest that the effects found here do not replicate, while technology readiness and education, for instance, could show to be important employee-related moderators affecting acceptability. Also in this study, average scores (independent from conditions) do not exceed neutral scores on employee acceptability, again urging for future work covering more modalities of implementation such as privacy management and measurement interpretation procedures. Because of this complexity in wearable MWL-monitoring acceptability and to raise ecological validity, we are additionally running a qualitative field-study involving operators working in two manufacturing companies. In this study, we link wearable MWL-monitoring to anonymity of data, organizational trust, autonomy-support and even human-robot collaboration (in terms of adaptive automation). We finally hope to see more contextualized qualitative and experimental work, being imperative to pinpoint the pivotal context-dependent and operator-dependent nuances in wearable MWLmonitoring.

\section{CONCLUSION}

The complexity of the Industry 4.0 workplace will bring about exciting opportunities, but also some key challenges for the future industrial operator. Wearable MWL-monitoring can help in addressing challenges to job-related mental workload as induced by top-notch technology such as augmented reality or collaborative robots. Still, the employee acceptability of wearable MWL-monitoring might be most pivotal in assuring its successful implementation. We found that framing this technology's end-goals as serving intrinsic goals within an autonomy-supportive work context yields higher employee acceptability as compared to framing its purposes in terms of serving extrinsic goals within a controlling work context. The former condition still did not result in an acceptability score exceeding neutrality. As more nuanced and specific scrutiny is highly needed to help in addressing this important societal challenge by helping to set out the boundary conditions and guidelines for application, the authors look forward to learn from future work iterating on the current findings.

\section{REFERENCES}

Alder, G.S. (2001), "Employee reactions to electronic performance monitoring: A consequence of organizational culture", The Journal of High Technology Management Research, Vol. 12 No. 2, pp. 323-342. http://dx.doi.org/10.1016/S1047-8310(01)00042-6

Baard, P.P., Deci, E.L. and Ryan, R.M. (2000), "Intrinsic need satisfaction as a motivational basis of performance and well-being at work", Unpublished Manuscript, Fordham University.

D’Addona, D.M., Bracco, F., Bettoni, A., Nishino, N., Carpanzano, E. and Bruzzone, A.A. (2018), “Adaptive automation and human factors in manufacturing: An experimental assessment for a cognitive approach", CIRP Annals, CIRP, Vol. 67 No. 1, pp. 455-458. http://dx.doi.org/10.1016/j.cirp.2018.04.123

Deci, E.L., Olafsen, A.H. and Ryan, R.M. (2017), "Self-Determination Theory in Work Organizations: The State of a Science”, Annual Review of Organizational Psychology and Organizational Behavior, Vol. 4 No. 1, pp. 19-43. http://dx.doi.org/10.1146/annurev-orgpsych-032516-113108

Deci, E.L. and Ryan, R.M. (2000), “The 'What' and 'Why' of Goal Pursuits: Human Needs and the SelfDetermination of Behavior”, Psychological Inquiry, Vol. 11 No. 4, pp. 227-268. https://doi.org/10.1207/S15327965PLI1104_01

Kasser, T. and Ryan, R.M. (1996), "Further Examining the American Dream: Differential Correlates of Intrinsic and Extrinsic Goals", Personality and Social Psychology Bulletin, Vol. 22 No. 3, pp. 280-287. https://doi.org/10.1177/0146167296223006

\footnotetext{
${ }^{1}$ The pre-registration for this follow-up study and all materials as well as for the pilot study presented here are available at https://osf.io/vhxku/?view_only=e5fca7f6c8244636a8160abbdc8c23bc.
} 
Longo, F., Nicoletti, L. and Padovano, A. (2017), "Smart operators in industry 4.0: A human-centered approach to enhance operators' capabilities and competencies within the new smart factory context", Computers and Industrial Engineering, Vol. 113, pp. 144-159. http://dx.doi.org/10.1016/j.cie.2017.09.016

Gao, Y., Li, H. and Luo, Y. (2015), "An empirical study of wearable technology acceptance in healthcare”, Industrial Management \& Data Systems, Vol. 115 No. 9, pp. 1704-1723. https://doi.org/10.1108/IMDS03-2015-0087

Matthews, G. (2016), "Multidimensional profiling of task stress states for human factors: A brief review", Human Factors: The Journal of the Human Factors and Ergonomics Society, Vol. 58 No. 6, pp. 801-813. http://dx.doi.org/10.1177/0018720816653688

Mitchell, J.I., Gagné, M., Beaudry, A. and Dyer, L. (2012), "The role of perceived organizational support, distributive justice and motivation in reactions to new information technology", Computers in Human Behavior, Vol. 28 No. 2, pp. 729-738. http://dx.doi.org/10.1016/j.chb.2011.11.021

Oreg, S. (2006), "Personality, context, and resistance to organizational change", European Journal of Work and Organizational Psychology, Vol. 15 No. 1, pp. 73-101. https://doi.org/10.1080/13594320500451247

Parasuraman, A. and Colby, C.L. (2015), “An Updated and Streamlined Technology Readiness Index: TRI 2.0”, Journal of Service Research, Vol. 18 No. 1, pp. 59-74. http://dx.doi.org/10.1177/1094670514539730

Prasad, J. and Agarwal, R. (1997), "The Role of Innovation Characteristics and Perceived Voluntariness in the Acceptance of Information Technologies", Decision Sciences, Vol. 28 No. 3, pp. 557-582. http://dx.doi.org/10.1111/j.1540-5915.1997.tb01322.x

Rigby, C.S. and Ryan, R.M. (2018), "Self-Determination Theory in Human Resource Development: New Directions and Practical Considerations", Advances in Developing Human Resources, Vol. 20 No. 2, pp. 133-147. http://dx.doi.org/10.1177/1523422318756954

Roche, M. and Haar, J.M. (2013), “A metamodel approach towards self-determination theory: A study of New Zealand managers' organisational citizenship behaviours”, International Journal of Human Resource Management, Vol. 24 No. 18, pp. 3397-3417. http://dx.doi.org/10.1080/09585192.2013.770779

Rupp, M.A., Michaelis, J.R., Mcconnell, D.S. and Smither, J.A. (2016), “The Impact of Technological Trust and Self-Determined Motivation on Intentions to use Wearable Fitness Technology; The Impact of Technological Trust and Self-Determined Motivation on Intentions to use Wearable Fitness Technology", Proceedings of the Human Factors and Ergonomics Society, pp. 1434-1438.

Ryan, R. and Deci, E. (2000), "Self-determination theory and the facilitation of intrinsic motivation", American Psychologist, Vol. 55 No. 1, pp. 68-78. http://dx.doi.org/10.1037/0003-066X.55.1.68

Sarpong, S. and Rees, D. (2014), "Assessing the effects of 'big brother' in a workplace: The case of WAST", European Management Journal, Vol. 32 No. 2, pp. 216-222. http://dx.doi.org/10.1016/j.emj.2013.06.008

Schall, M.C., Sesek, R.F. and Cavuoto, L.A. (2018), "Barriers to the Adoption of Wearable Sensors in the Workplace: A Survey of Occupational Safety and Health Professionals”, Human Factors, Vol. 60 No. 3, pp. 351-362. http://dx.doi.org/10.1177/0018720817753907

Sparrow, P.R. (2001), "Developing diagnostics for high performance organization cultures", in Cooper, C.L., Cartwright, S. and Earley, P.C. (Eds.), The International Handbook of Organizational Culture and Climate, Wiley, Chichester, pp. 85-106.

Van Acker, B.B., Parmentier, D.D., Vlerick, P. and Saldien, J. (2018), "Understanding mental workload: From a clarifying concept analysis toward an implementable framework", Cognition, Technology \& Work, Springer, London, Vol. 20 No. 3, pp. 351-365. http://dx.doi.org/10.1007/s10111-018-0481-3

Van den Broeck, A., Van Ruysseveldt, J., Smulders, P. and De Witte, H. (2011), "Does an intrinsic work value orientation strengthen the impact of job resources? A perspective from the Job Demands-Resources Model”, European Journal of Work and Organizational Psychology, Vol. 20 No. 5, pp. 581-609. http://dx.doi.org/10.1080/13594321003669053

Vansteenkiste, M., Neyrinck, B., Niemiec, C.P., Soenens, B., Witte, H. and Broeck, A. (2007), "On the relations among work value orientations, psychological need satisfaction and job outcomes: A self-determination theory approach", Journal of Occupational and Organizational Psychology, Vol. 80 No. 2, pp. 251-277. http://dx.doi.org/10.1348/096317906X111024

Vansteenkiste, M., Simons, J., Lens, W., Sheldon, K.M. and Deci, E.L. (2004), "Motivating learning, performance, and persistence: The synergistic effects of intrinsic goal contents and autonomy-supportive contexts", Journal of Personality and Social Psychology, Vol. 87 No. 2, pp. 246-260. http://dx.doi.org/10.1037/0022-3514.87.2.246

Vansteenkiste, M., Simons, J., Lens, W., Soenens, B. and Matos, L. (2005), "Examining the impact of intrinsic versus intrinsic goal framing and internally controlling versus autonomy-supportive communication style upon early adolescents academic achievement", Child Development, Vol. 76 No. 2, pp. 483-501. http://dx.doi.org/10.1111/j.1467-8624.2005.00858.x

Venkatesh, V. and Davis, F.D. (2000), “A Theoretical Extension of the Technology Acceptance Model: Four Longitudinal Field Studies”, Management Science, Vol. 46 No. 2, pp. 186-204.

https://doi.org/10.1287/mnsc.46.2.186.11926 
Vlassenroot, S., Brookhuis, K., Marchau, V. and Witlox, F. (2010), “Towards defining a unified concept for the acceptability of Intelligent Transport Systems (ITS): A conceptual analysis based on the case of Intelligent Speed Adaptation (ISA)", Transportation Research Part F: Traffic Psychology and Behaviour, Vol. 13 No. 3, pp. 164-178. http://dx.doi.org/10.1016/j.trf.2010.02.001

Watkins Allen, M., Walker, K.L., Coopman, S.J. and Hart, J.L. (2007), "Workplace surveillance and privacy", Management Communication Quarterly, Vol. 21 No. 2, pp. 172-200. http://dx.doi.org/10.1177/0893318907306033

Wickens, C.D. (2017), "Mental workload: assessment, prediction and consequences”, in Longo, L. and Leva, M.C. (Eds.), Human Mental Workload: Models and Applications: First International Symposium, $H$ WORKLOAD 2017, Dublin, Ireland, June 28-30, 2017, Revised Selected Papers, Springer International Publishing, Cham, pp. 18-29.

Young, M., Brookhuis, K., Wickens, C. and Hancock, P. (2014), "State of science: Mental workload in ergonomics", Ergonomics, Vol. 58 No. 1, pp. 1-17. http://dx.doi.org/10.1080/00140139.2014.956151

\section{ACKNOWLEDGMENTS}

This work was funded by Flanders Make as part of the SBO project Yves, Oude Diestersebaan, 133, 3920 Lommel, Belgium. 
